\title{
Morphological and molecular data confirm the transfer of homostylous species in the typically distylous genus Galianthe (Rubiaceae), and the description of the new species Galianthe vasquezii from Peru and Colombia
}

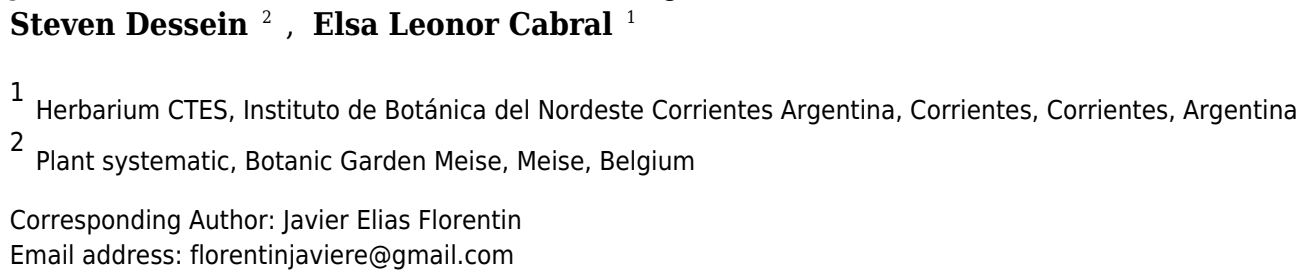

Galianthe (Rubiaceae) is a neotropical genus comprising 50 species divided into two subgenera, Galianthe subgen. Galianthe, with 39 species and Galianthe subgen. Ebelia, with 11 species. The diagnostic features of the genus are: usually erect habit with xylopodium, distylous flowers arranged in lax thyrsoid inflorescences, bifid stigmas, 2carpellate and longitudinally dehiscent fruits, with dehiscent valves or indehiscent mericarps, plump seeds or complanate with a wing-like strophiole, and pollen with double reticulum, rarely with a simple reticulum. This study focused on two species that were originally described under Diodia due to the occurrence of fruits indehiscent mericarps: Diodia palustris and D. spicata. In the present study, classical taxonomy is combined with molecular analyses. As a result, we propose that both Diodia species belong to Galianthe subgen. Ebelia. The molecular position within Galianthe, based on ITS and ETS sequences, has been supported by the following morphological characters: thyrsoid, spiciform or cymoidal inflorescences, bifid stigmas, pollen grains with a double reticulum, and indehiscent mericarps. However, both species, unlike the remainder of the genus Galianthe, have homostylous flowers, so the presence of this type of flower significantly modifies the generic concept. In this framework, a third homostylous species, Galianthe vasquezii, from the Andean region is also described. Until now, this species remained cryptic under specimens of Galianthe palustris It differs however from the latter by having longer calyx lobes, the presence of dispersed trichomes inside the corolla lobes (vs. glabrous), fruits that are acropetally dehiscent (vs. basipetally dehiscent), and its Andean geographical distribution (vs. Paranaense). Additionally, a lectotype has been chosen for Diodia palustris, Borreria pterophora has been placed under synonymy of Galianthe palustris, and Galianthe boliviana is reported for the first time from Peru. A key of all 
Galianthe species with indehiscent mericarps is also provided. 
1 Morphological and molecular data confirm the transfer of homostylous species in the

2 typically distylous genus Galianthe (Rubiaceae), and the description of the new species

3 Galianthe vasquezii from Peru and Colombia

4 Javier E. Florentín ${ }^{1}$, Andrea A. Cabaña Fader ${ }^{1}$, Roberto M. Salas ${ }^{1}$, Steven Janssens ${ }^{2}$, Steven

5 Dessein $^{2}$ and Elsa Cabral ${ }^{1,}$

$6{ }^{1}$ Herbarium CTES, Instituto de Botánica del Nordeste, Corrientes, Argentina

$7 \quad$ 2Plant systematic, Botanic Garden Meise, Meise, Belgium

8 Author for correspondence:

9 Javier E. Florentín 1

10 Email address: florentinjaviere@gmail.com

11 ABSTRACT

Galianthe (Rubiaceae) is a neotropical genus comprising 50 species divided into two subgenera, Galianthe subgen. Galianthe, with 39 species and Galianthe subgen. Ebelia, with 11 species. The diagnostic features of the genus are: usually erect habit with xylopodium, distylous flowers arranged in lax thyrsoid inflorescences, bifid stigmas, 2-carpellate and longitudinally dehiscent fruits, with dehiscent valves or indehiscent mericarps, plump seeds or complanate with a winglike strophiole, and pollen with double reticulum, rarely with a simple reticulum. This study focused on two species that were originally described under Diodia due to the occurrence of fruits indehiscent mericarps: Diodia palustris and D. spicata. In the present study, classical taxonomy is combined with molecular analyses. As a result, we propose that both Diodia species belong to Galianthe subgen. Ebelia. The molecular position within Galianthe, based on ITS and ETS sequences, has been supported by the following morphological characters: thyrsoid, spiciform or cymoidal inflorescences, bifid stigmas, pollen grains with a double reticulum, and indehiscent mericarps. However, both species, unlike the remainder of the genus Galianthe, have homostylous flowers, so the presence of this type of flower significantly modifies the generic concept. In this framework, a third homostylous species, Galianthe vasquezii, from the Andean region is also described. Until now, this species remained cryptic under specimens of Galianthe palustris It differs however from the latter by having longer calyx lobes, the presence of dispersed trichomes inside the corolla lobes (vs. glabrous), fruits that are acropetally dehiscent (vs. basipetally dehiscent), and its Andean geographical distribution (vs. Paranaense). Additionally, a lectotype has been chosen for Diodia palustris, Borreria pterophora has been 
placed under synonymy of Galianthe palustris, and Galianthe boliviana is reported for the first time from Peru. A key of all Galianthe species with indehiscent mericarps is also provided.

\section{INTRODUCTION}

Galianthe Griseb. is a neotropical genus belonging to tribe Spermacoceae (Groeninckx et al., 2009a). The genus was revised by Cabral (2002) and divided into two subgenera (Cabral \& Bacigalupo, 1997): Galianthe subgen. Galianthe, from South America with 39 species, and Galianthe subgen. Ebelia (Rchb.) E.L. Cabral \& Bacigalupo, with 11 Mesoamerican and South American species. Historically, Galianthe was associated with Diodia L., which has been described based on only D. virginiana L. The type species has a palustrine habit, pauciflorous axillary inflorescences, filiform corolla tube, bifid style with two long filiform stigmatic lobes, and indehiscent fruits. Later, others authors (i.e. Swartz 1788; Walter 1788, Candolle 1830, Small 1913) added other species into this genus with diverse kinds of habits and inflorescences, different floral morphology (e.g. distyly or homostyly, infundibuliform or campanulate corollas, bifid or bilobate stigma), and 2-carpellate schizocarpic fruits, being currently comprised by ca. 180 names (called Diodia s. lat.). Later, Bacigalupo \& Cabral (1999) revised the genus Diodia and maintained only five species that were morphologically similar to the type species $D$. virginiana L. (description as above, and constituting Diodia s. str.). Species that did not match with these diagnostic features, were transferred to other genera as follows: eight species to Borreria subgen. Dasycephala (DC.) Bacigalupo \& E.L. Cabral (Bacigalupo \& Cabral, 1996), 12 species to Hexasepalum Bartl. ex DC. (Kirkbride 2014; Kirkbride \& Delprete, 2015; Cabaña Fader et al., 2016), and 4 species to Galianthe subgen. Ebelia (Cabral \& Bacigalupo, 1997). The four Galianthe species are distylous, Diodia bogotensis (Kunth) Cham. \& Schltdl. [= Galianthe bogotensis (Kunth) E.L. Cabral \& Bacigalupo]; D. brasiliensis Spreng. [= G. brasiliensis (Spreng.) E.L. Cabral \& Bacigalupo]; D. cymosa Cham. [=G. cymosa (Cham.) E.L. Cabral \& Bacigalupo], and D. hispidula A. Rich. ex DC. [=G. hispidula (A. Rich. ex DC.) E.L. Cabral \& Bacigalupo]. The remaining species with an uncertain position (ca. 150 names) are currently under revision (Cabaña Fader com. pers.). In this sense, Bacigalupo and Cabral (1996, 1998) transferred these species to Borreria subgen. Dasycephala because of their homostylous flowers and indehiscent mericarps, while Delprete et al. (2005) and Delprete (2007), alluding to a broad concept, transferred the two species to Spermacoce mainly based on fruit characters. Dessein (2003) informally proposed to consider Diodia spicata as part of Galianthe based on molecular data (ITS intron), palynological data (double reticulum), and fruit morphology. The aim of this 
64 work is to confirm the taxonomic position of D. palustris and D. spicata based on morphological

65 and molecular data, and perform their formal combination in Galianthe. In addition, a third

66 homostylous species (Galianthe vasquezii R.M Salas \& J. Florentín) is described and illustrated

67 based on specimens from Colombia and Peru (previously identified as D. palustris). Additionally,

68 a lectotype has been chosen for Diodia palustris whereas Borreria pterophora has been placed

69 under synonymy of Galianthe palustris. Moreover Galianthe boliviana E.L. Cabral is for the

70 first time recorded in Peru. Finally, we provided a distribution map for the species investigated in

71 this study, as well as a dichotomous key for all taxa with indehiscent mericarps.

\section{MATERIALS AND METHODS}

\section{Morphological Study}

This study is based on classical taxonomy techniques. Collections deposited at the BA, BHCB, CEPEC, CTES, ESA, FUEL, FPS, FURB, HAS, HOXA, HUT, IAC, IAN, IFFSC, IPA, K, LIL, MBM, MO, NY, P, PR, SI, SP, UB, UFRN, USB, US, USM and UEC herbaria were analysed. Furthermore, the databases of the 'Catálogo de plantas e fungos do Brazil' and 'Missouri Botanical Garden' were examined. In order to carry out scanning electron microscope (SEM) analyses, flowers were dehydrated using a graded series of ethanol solutions and afterwards critically point dried and sputter-coated with gold-palladium. SEM images were obtained with a JEOL 5800 LV scanning electron microscope. Pollen grains were acetolyzed according to Erdtman (1966) and mounted in glycerine jelly for analysis by light microscopy (LM). Conventional parameters ( $\mathrm{P}=$ polar axis, $\mathrm{E}=$ equatorial axis) of at least 20 grains were measured under LM, and the exine was analyzed using SEM. Pollen terminology follows Punt et al. (2007). Species distribution maps were generated from distribution data that was present on the herbarium labels for each specimen and subsequently georeferenced using Google Earth (2015) and DIVA-GIS (2013).

"The electronic version of this article in Portable Document Format (PDF) will represent a published work according to the International Code of Nomenclature for algae, fungi, and plants (ICN), and hence the new names contained in the electronic version are effectively published under that Code from the electronic edition alone. In addition, new names contained in this work which have been issued with identifiers by IPNI will eventually be made available to the Global Names Index. The IPNI LSIDs can be resolved and the associated information viewed through any standard web browser by appending the LSID contained in this publication to the prefix 
95 "http://ipni.org/". The online version of this work is archived and available from the following

96 digital repositories: PeerJ, PubMed Central, and CLOCKSS".

97 Molecular study

98 In total, 45 species (47 accessions) were included to infer the phylogenetic relationship of Diodia 99 palustris and D. spicata. The ingroup contains species from the Borreria, Carajasia R.M. Salas, 100 E.L. Cabral \& Dessein, Crusea Cham. \& Schltdl., Diodia, Emmeorhiza Pohl ex Endl., Ernodea 101 Sw., Galianthe, Hexasepalum, Mitracarpus Zucc., Psyllocarpus Mart. \& Zucc., Richardia L., 102 Schwendenera K. Schum., Spermacoce, and Staelia Cham. \& Schldtl. genera, and Bouvardia 103 ternifolia (Cav.) Schltdl. as the outgroup. Leaf samples of these studies were obtained from silica 104 gel-dried material or herbarium materials. Forty-three species (44 accessions) were previously 105 used by Salas et al. (2015). Four accessions belonging to D. palustris has been added. All studied species with geographical information, collector, herbarium and GenBank accession numbers are 107 provided in Appendix.

108 Molecular Protocols

109 Total genomic DNA was isolated from silica-dried leaf material using a modified CTAB protocol 110 (Doyle and Doyle 1987). Nuclear ribosomal ETS and ITS fragments were amplified following 111 Baldwin and Markos (1998) and NegrónOrtiz and Watson (2002), and White et al. (1990), 112 respectively. PCR reactions for both gene markers investigated in this study consisted of 2 min 113 initial denaturation at $94{ }^{\circ} \mathrm{C}$ and 30 cycles of $30 \mathrm{~s}$ denaturation at $94{ }^{\circ} \mathrm{C}, 30 \mathrm{~s}$ primer annealing at 114 primer specific temperature and 1 min extension at $72{ }^{\circ} \mathrm{C}$. Primer annealing for ETS and ITS 115 were at $47^{\circ} \mathrm{C}$ and $48^{\circ} \mathrm{C}$ respectively. Amplification reactions were carried out on a GeneAmp 116 PCR system 9700 (Applied Biosystems). Purified amplification products were sent to Macrogen, 117 Inc. (Seoul, South Korea) for sequencing. Sequences obtained in this study were deposited at 118 GenBank [Diodia palustris, Verdi et al. 1905, ETS (MF166824), ITS (MF166826); Miguel et al. 19, ETS (MF166825), ITS (MF166827).

\section{Phylogenetic analyses}

121 Contiguous sequences were assembled using Geneious v7.0.6 (Biomatters, New Zealand). 122 Automatic alignments were carried out with MAFFT (Katoh et al., 2002) Subsequent manual 123 finetuning of the aligned dataset was done in Geneious v7.0.6. Congruency between the different datasets was inferred using different methods. First, a series of incongruence length difference 
125 tests (ILD; Farris et al., 1995) were carried out with PAUP* v.4. 0b10 (Swofford, 2003) using the

126 following parameters: simple taxon addition, TBR branch swapping and heuristic searches of 1271000 repartitions of the data. Despite the well-known sensitivity of the ILD test (Barker and 128 Lutzoni, 2002), the results of this test were compared in light of the resolution and support values 129 of the obtained nuclear and nuclear ribosomal topologies. As a result, possible conflict between 130 data matrices was visually inspected, searching for conflicting relationships within each topology 131 that are strongly supported (hard vs. soft incongruence; Johnson and Soltis, 1998). Model 132 selection for the Bayesian inference analysis was conducted with ModelTest 3.06 (Posada \& 133 Crandall 1998) under the Akaike Information Criterium (AIC). The GTR + G model was selected 134 for both ITS and ETS. Bayesian analyses of the concatenated dataset were carried out with 135 MrBayes 3.1 (Huelsenbeck \& Ronquist 2001, Ronquist \& Huelsenbeck 2003). Four chains (one 136 cold, three heated), initiated from a random starting tree were run simultaneously for 10 million generations. Every 1000 generations, a tree was sampled from the chain for a total of 10,000 trees. Due to the burn-in, $50 \%$ of the sample points were discarded. Convergence of the chains was examined with TRACER 1.4 (Rambaut \& Drummond 2007). This resulted in an effective sampling size (ESS) parameter exceeding 100, which assumes a sufficient sampling and acceptable mixing.

\section{RESULTS}

\section{Phylogenetic results}

144 The ingroup contains 14 genera represented by 45 species of the Spermacoce clade. Of these, Diodia spicata and D. palustris are analysed for the first time in this context. ITS and ETS datasets were analysed both separately and combined. Because topology of each gene marker is very similar, we only present the results of the combined analysis (Fig. 1). Current results indicate that most clades coincide with most currently accepted genera (e.g. Crusea, Emmeorhiza, Ernodea, Diodia s.s. (sensu Bacigalupo \& Cabral, 1999), Mitracarpus, Psyllocarpus, Richardia and Staelia). Spermacoce, Borreria and Hexasepalum however, appear unresolved or as polyphyletic genera being present in several different parts of the tree. In regard of the species studied, we found that G. spicata and G. palustris fall intermingled among the Galianthe species. The Galianthe clade, including both former Diodia species, is strongly supported (Bayesian Posterior Probability (BBP): 100). The genus is divided into two strongly 
156 (Cham. \& Schltdl.) E.L. Cabral, and G. peruviana (Pers.) E.L. Cabral (BPP: 100), all from G. 157 subgen. Galianthe. The other subclade (BPP: 99) comprises G. spicata, G. palustris, and G. 158 brasiliensis (type species of Galianthe subgen. Ebelia). The clade of Galianthe and Carajasia is 159 only moderately supported (BBP: 79). The genera Galianthe and Carajasia share the occurrence 160 of pollen grains with a double reticulum, mostly associated with the distyly and bifid stigma. The 161 Galianthe-Carajasia clade forms an unsupported trichotomy with Schwendenera (also distylous) 162 and the remaining genera of the Spermacoce clade (all homostylous species never associated to 163 double reticulum pollen grains). Interestingly, all clades that coincide with generic concepts are 164 strongly supported [e.g. Psyllocarpus (BBP:99), Spermacoce s.s. (BBP:100), Richardia 165 (BBP:100), Borreria s.s. (BBP:100), Mitracarpus (BBP:97), Hexasepalum s.s., Staelia (BBP:99), 166 Diodia s.s. (BBP:100), Borreria latifolia group (BBP:100), and Crusea (BBP:100)]. The species 167 assigned to Borreria (sensu Bacigalupo \& Cabral, 1996) are divided into two clades that are 168 intermingled with other morphologically well-defined genera. One of these clades, further referred to as the Borreria latifolia group, comprises Borreria alata, B. schumannii, and B. latifolia (BBP:100). The other clade comprises five Borreria species from North and South America (B. capitata, B. multibracteata, B. tenella, B. dasycephala, and B. verticillata), as well as the African Spermacoce dibrachiata (BBP: 100). Spermacoce is divided into two unrelated branches, of which one clade comprises the type species $S$. tenuior, other American species with similar flower morphology (S. eryngioides, S. prostrata, S. incognita, S. confusa, and S. glabra, all with stamens and style included), and the Australian S. breviflora (support 100). As mentioned above, the other species of Spermacoce (S. dibrachiata) falls among the species of Borreria. Hexasepalum species are also divided into two clades, one of them is well supported (BBP: 99) and contain H. angustifolium Bart. ex DC. (type species), H. apiculatum and H. teres. The other, only represented by $H$. sarmentosum appears as sister species of the Ernodea (BBP: 100). The genus Ernodea, represented by E. taylori and E. littoralis, constitutes a strongly supported clade (BBP: 100). The results explained above allow us to support the following taxonomic changes.

TAXONOMIC TREATMENT

\section{Description of the new species}

184 Galianthe vasquezii R. M. Salas \& J. Florentín, sp. nov. TYPE. PERU: Pasco, Oxapampa, 185 Parque Nacional Yanachaga-Chemillen, Quebrada Yanachaga, $2250 \mathrm{~m}, 1^{\circ} 24^{\prime} \mathrm{S}, 7^{\circ} 28^{\prime} \mathrm{W}, 14 \mathrm{Jun}$ 186 2003, R. Vásquez M. 28284 (holotype: HOXA!; isotypes MO!, HUT, USM). 
187

188

189

190

191

192

193

194

195

196

197

198

199

200

201

202

203

204

205

206

207

208

209

210

211

212

213

214

215

216

\section{Description}

Herb decumbent or prostrate, stems quadrangular, angle strongly alate, with scabridous papillae, more densely disposed near nodes. Leaves sessile or pseudopetiolate, pseudopetiole up to $4 \mathrm{~mm}$ long, blades elliptic or obovate, apex acute, base attenuate, 12-32 $\times 5-17 \mathrm{~mm}$, plicate-nervose, adaxially glabrous or puberulous, abaxially scabridous on nerves, margin scabridous, with 3-5 secondary nerves; stipular sheath 3.2-5.6 mm long, with 7-9 linear fimbriae, glabrous, fimbriae 3.5-6.8 mm long. Inflorescences thyrsoid, partial inflorescences subglomeriform, multiflorous. Flower pedicellate; pedicel 1-2 mm long; calyx (3-) 4-lobed, hypanthium 1.1-1.3 mm long, glabrous or glabrescent, lobes narrowly triangular, 1-1.4 mm long, glabrous, apex acute; corolla infundibuliform, 3-lobed, 1.75-2.1 mm long, white; lobes ovate, internally with hairs scattered at base, tube internally with some dispersed hairs near its base and externally glabrous, straight; stamens subincluded, anther 0.4-0.6 mm long, oblong, filament fixed immediately below interlobular sinuses; pollen grains 7-8 zonocolpate, oblate-spheroidal to prolate spheroidal, small, $\mathrm{P}=31 \mu \mathrm{m}, \mathrm{E}=29 \mu \mathrm{m}$, colpi long, endoaperture an endocingulum, exine semitectate, reticulate, muri nanospinose, 0,18-0,3 $\mu \mathrm{m}$ long; style bifid, 1.5-1.8 mm long, stigmatic branches ca. $0.2 \mathrm{~mm}$ long, with conspicuous papillae. Fruit a capsule, cordate or deltoid in outline, 1.8-2 × 1.6-1.9 $\mathrm{mm}$, glabrous, with two indehiscent mericarps which split from the base upwards, each valve remains temporary attached in upper half, at maturity caduceus, seed 1.8-2 $\times 0.8-1 \mathrm{~mm}$, ovoid, ventral face longitudinally furrowed, partially covered by the strophiole; exotesta reticulatefoveate. Fig. 2, 3

Distribution-Andes of Peru and Colombia.

Observations-All specimens of the G. vasquezii were previously identified as Galianthe palustris. However, it differs from G. palustris, in having calyx lobes 1-1.4 mm long, with acute apex (vs. calyx lobes 0.4-0.6 mm long, obtuse), corolla 1.75-2.1 mm long, lobes internally with hairs scattered at base, tube internally with some dispersed hairs near its base (vs. corolla 1-1.5 $\mathrm{mm}$ long, internally glabrous), pollen grains with reticulate exine, muri nanospinose (vs. pollen grains with bireticulate exine, suprareticulum psilate and incomplete, infrareticulum nanospinose), fruit 1.8-2. mm long, deltoid in outline, acropetally dehiscent (vs. fruit 1.1-1.5 mm long, oblong or obovate in outline, basipetally dehiscent), and seeds 1.8-2 mm long (vs. seeds 1$1.42 \mathrm{~mm}$ long). 
217 Ecology - Galianthe vasquezii grows in Montane Forest of Peru and Colombia, which represents 218 a severely fragmented type of vegetation. It grows between 1800 and $2500 \mathrm{~m}$ altitude.

219 Conservation status - The extent of occurrence (EOO) was calculated to be $397 \mathrm{~km}^{2}$ (cell sized 2 $220 \mathrm{~km}$ ). Following the IUCN criteria (IUCN, 2001), this species should be considered as endangered 221 [EN B1 ab (ii, iii)], due to the continuing decline in area and quality of its habitat.

Additional Specimens Examined-COLOMBIA: Antioquia, Monte del Diablo, 21 Jul. 1944, 223 Bro. Daniel 3303 (US); Rio Negro, 16 Dec. 1933, Bro. Daniel 165 (US). PERU: Distrito 224 Huancabamba, sector Grapanazú, límite Parque Nacional, Yanachaga-Chemillen, 10²6'S, $75^{\circ} 23^{\prime} \mathrm{W}, 15$ Oct. 2003, R. Rojas et al. 1892 (MO); idem, sector Tunqui, camino hacia María Puñis, $1895 \mathrm{~m}, 1^{\circ} 16^{\prime} 31^{\prime \prime S}, 7^{\circ} 30^{\prime} 59^{\prime \prime} \mathrm{W}$, M. Cueva 193 (HOXA, HUT, MO, USM); Luispicanchi, Cuzco, Quincemil, 13²14'S, 7045'W, Oct. 1950, F. Marín 2731 (CTES, LIL);

Pasco, Oxapampa, carretera de Cochabamba, 10³3'42"S, 75²7'23"W, 11 Nov. 2004, $A$. Monteagudo et al. 7587 (CTES, MO).

New combinations

Galianthe palustris (Cham. \& Schltdl.) Cabaña Fader \& E. L. Cabral, comb. nov. Diodia palustris Cham. \& Schltdl., Linnaea 3: 347. 1828. Borreria palustris (Cham. \& Schltdl.) Bacigalupo \& E. L. Cabral, Hickenia 2: 264. 1998. Spermacoce palustris (Cham. \& Schltdl.) Delprete, Fl. Il. Catarin. (2): 740. 2005. TYPE: BRAZIL, Santa Catarina, "Ad fretum St. Catharinae Brasiliae ipsi legimus, in palustribus Brasiliae aequinoctiales", s. d., F. Sellow s.n. (holotype: B destroyed, lectotype here designed PR!).

Borreria gymnocephala DC., Prodr. 4: 549. 1830. Diodia gymnocephala (DC.) K. Schum., in

Borreria pterophora C. Presl., Abh. Königl. Böhm. Ges. Wiss. V, 3: 516. 1845. nov. syn. TYPE: BRAZIL, Rio Janeiro, s.1., s.d., Beske s.n. (holotype: PR!).

241 Diodia alata Nees \& Mart., Nova Acta Acad. Caes. Leop. Carol., Wied-Neuwied 12: 12.1824.

242 Dasycephala alata (Nees \& Mart.) Benth. \& Hook. f. ex B.D. Jacks, Index Kew. 2: 719. 1893.

243 TYPE: BRAZIL, s.1., s.d., M. Wied s.n. (holotype BR!; isotypes: LD, LE, W!).

244 Diodia microcarpa K. Schum. ex Glaz., Bull. Soc. Bot. France 56 (Mém. 3d): 361. 1909. TYPE:

245 BRAZIL, “Brasília”, A.F.M. Glaziou 18283 (holotype B destroyed, photo F 867!). 
246

247

248

249

250

251

252

253

254

255

256

257

258

259

260

261

262

263

264

265

266

267

268

269

270

271

272

273

274

275

276

\section{Description}

Herb stoloniferous, sometimes with ascendant stems. Stems quadrangular, angle strongly winged, wing with long and slightly recurved fimbriae, or with scabridous and retrorse papillae, especially near foliar nodes. Leaves subsessile or pseudopetiolate; pseudopetiole 1-4 mm long; blades elliptic or obovate, rarely orbicular, $22-30 \mathrm{~mm} \times 10-18.6 \mathrm{~mm}$, apex obtuse, acute or acuminate, base attenuate, plicate-nervose, with 3-4 secondary nerves, adaxially glabrous or scaberulous, margin scaberulous, abaxially scabridous only on nerves; stipular sheath 3-4 mm long, basally alate, 7-9 fimbriate, fimbriae linear, glabrous, 5-8 $\mathrm{mm}$ long. Inflorescences thyrsoid, partial inflorescences congested and multiflorous, sometimes arranged on pleiochasium. Flowers shortly pedicellate; pedicel 0.5-1 mm long; calyx 2(-3)-lobed, hypanthium glabrous, lobes triangular, apex obtuse, succulent, 0.4-0.6 mm long; corolla 2-3-lobed, infundibuliform, white, 1-1.5 mm long, tube internally and externally glabrous, scarcely papillate on apex of the dorsal face of lobes; stamens 2-3, anthers 0.2-0.34 mm long, oblong, dorsal surface with a bullate connective, immediately above the insertion of the filament; pollen grains 6-7(-8) zonocolporate, oblatespheroidal, small, $\mathrm{P}=24 \mu \mathrm{m}, \mathrm{E}=25.3 \mu \mathrm{m}$, long colpi, endoaperture an endocingulum, exine bireticulate, suprareticulum incomplete, muri psilate, infrareticulum complete, muri nanospinose, 0,15-0,28 $\mu \mathrm{m}$ long; style bifid, 1-1.5 mm long, stigmatic branches 0.2-0.46 mm long, notoriously papillate. Fruit a capsule, which separates from the apex downwards into two indehiscent mericarps, both mericarps remain basally united to the pedicel, tardily deciduous, oblong or ovate in outline, glabrous; seeds 1-1.42 $\times$ 0.7-0.8 mm, ovate or obpiriforme in outline, ventral surface with a longitudinal furrow covered by a persistent strophiole; exotesta reticulate-foveate. Fig. 3

Distribution-Brazil (Bahia, Minas Gerais, Paraná, Rio de Janeiro, Rio Grande do Sul, Santa Catarina, and São Paulo), and Argentina, Misiones province.

Ecology-Galianthe palustris is a heliophilous plant that inhabits in swampy areas near lotic water bodies, especially along main rivers and their tributaries.

Additional Specimens Examined-ARGENTINA. Misiones: San Pedro, Parque Provincial Moconá, embarcadero, 7 Mar. 2013, M. D. Judkevich et al. 46 (CTES); idem, borde de arroyo, 11 Dec. 2011, L. M. Miguel et al. 19 (CTES). BRAZIL. Bahia: Belmonte, 23 Nov. 1970, T. S. Santos 1124 (CEPEC); Minas Gerais: Camanducaia, Monte Verde, Estrada Camanducaia, 27 Apr. 2013, J. A. M. Carmo 125 (UEC); ídem, Monte Verde, 24 Jan. 2013, J. A. M. Carmo 111 (UEC); ídem, Mata dos Vargas, 22 Mar. 2000, R. B Torres et al. 1176 (FUEL); Santos Dumont, s. d., H.L.M. 
277

278

279

280

281

282

283

284

285

286

287

288

289

290

291

292

293

294

295

296

297

298

299

300

301

302

303

304

305

306

307

308

309

Barreto 11339 (BHCB); São João do Manhuaçu, 19 km S of the intersection of Highway BR-116 \& BR-262, just N of the village of São João do Manhuaçu, 27 Mar. 1976, G. Davidse \& W. G. D'Arcy 11434 (SP). Paraná: Mun. Bocaiúva do Sul, Serra da Bocaína, 31 Mar. 2001, E. Barbosa et al. 654 (CTES, ESA, MBM); Serra de São Luís, BR 277, 19 Jan. 1985, M.S. Ferrucci et al. 284 (CTES); Fazenda Reserva, 85 Km SW of Guarapuava, on bank of brook near Barbaquá, 17 Mar. 1967, J. C. Lindeman et al. 4959 (CTES, MBM, NY, UB); Mun. Morretes, Serra Morumbi, picada ao Olimpo, 19 Jan. 1995, O. S. Ribas et al. 761 (CTES); Mun. Piraquara, Floresta, 9 Mar. 1947, G. Hatschbach 640 (CTES, LIL); BR-476, 7 Km E de Contendas, 26 Jan. 1985, A. Krapovickas \& C. L. Cristóbal 39632 (CTES); Fazenda de J. Rickli near Turvo, $40 \mathrm{~km} \mathrm{~N}$ of Guarapuava, forest, 9 May. 1967, J. C. Lindeman et al. 5280 (CTES); Curitiba, 17 May. 2002, J. Cordeiro 2233 (ESA). Rio Grande do Sul: Barracão, Parque Estadual de Espigão Alto, 1 Mar. 2001, M. Sobral \& J. Larocca s.n.(FURB); Capivari, Viamão, 15 Mar. 1975, Porto et al. 1389 (CTES); Esteio, 23 Mar. 1949, B. Rambo 40638 (LIL); Leopoldo, on Monte Jacaré, 7 Dec. 1948, B. Rambo 38588 (LIL); Pareci, Prope Montenegro, 31 Mar. 1950, B. Rambo 46536 (CTES); Porto Alegre, 17 Dec. 1932, B. Rambo s.n. (P04541549); idem, Morro da Gloria, 16 Dec. 1931, B. Rambo 577 (LIL); San Salvador, 14 Mar. 1947, A. Sehnem 2676 (SI); ídem, 16 Dec. 1933, B. Rambo 577 (SP); idem, Montenegro, 1 Mar. 1950, A. Sehnem 4426 (SI); Santana, 6 Apr. 1974, M.C. Sidia 27 (HAS, CTES). Rio de Janeiro: $17 \mathrm{Km}$ from praça da Parati on road from Parati to Cunha, 26 Apr. 1972, J. H. kirkbride 1729 (US); Nova Friburgo, 12 Nov. 1890, A. Glaziou 18283 (P02088844); Petrópolis, vale Bonsucesso, 13 Apr. 1968, B.D. Sucre 2738 (US); Serra da Mantiqueira, Maciço do Itatiaia, Parque Nacional do Itatiaia, 16 Apr. 1971, I. Gottsberger et al. 110 (CTES); idem, I. Gottsberger 110-16471(CTES); Serra dos Orgãos, 11 Jan. 1905, G. Gardner 445 (US). Santa Catarina: 6.5 KM NW de Aguas Mornas, caminho a Lourdes, 6 Feb. 1994, A. Krapovickas et al. 44793 (CTES); Am Wege in del Velha bei Blumenau, Oct.1888, E. H. G. Ule 1062 (US); Fazenda Farofa, trilha da estrada do meio, 6 Apr. 2007, R. P. M., Souza 103 (ESA); Pilões, Palhoça, 6 Apr. 1956, R. Reitz et al. 2997 (US, NY); Santa Terezinha, Urubici, 7 Apr. 2009, M. Verdi et al. 1905 (IFFSC); São Bento do Sul, Rio Natal. Estrada rumo ao Xikavitska (Salto Seco), 19 Feb. 2011, F. S. Meyer 982 (UFRN); São Bento do Sul, Trilha do Parque 23 de September, 14 Dec. 2014, P. Schwirkowski 732 (FPS); Taió, Fazenda Tarumã, 18 Feb. 2010, A. Korte \& A. Kniess 1821 (FURB); Três Barras, Guruvá, San Francisco do Sul, 7 Nov. 1957, R. Reitz et al. 5621 (NY, US); Urubici, Santa Terezinha, 7 Apr. 2009, M. Verdi et al. 1905 (CTES); São Paulo: Barra do Turvo, 24 Mar. 2005, M. Carboni, 110 (ESA); 10 km de Barra do Turvo em direção a Pariquera-Açu, 14 Feb. 1995, J. P. Souza, et al. 96 (SP); Boracéia, 26 Mar. 
310

311

312

313

314

315

316

317

318

319

320

321

322

323

324

1940, N.G. Blanco s. n. (SP); Campinas, Lago próximo ao parque ecológico da UNICAMP, 1 Jun. 1995, L. Y. S Aona \& A. D. Faria 95/50 (SP); Campos das Sete Lagôas, Fazenda Campininha, just north of Rio Mogi-Guaçu 1,8 km NW of Pádua Sales, Mogi Guaçu, 4 Dec. 1961, G. Eiten 3517 (SP); Cananéia, Serra do Tambor, Vale do Ribeira, sul do Estado de São Paulo, 20 Nov. 2006, M. A Pinho-Ferreira et al. 673 (UEC); Cunha, Trilha do Rio Bonito, Parque Estadual da Serra do Mar, 19 Mar. 1996, A. Rapini et al. 73 (UEC); Cunha, Parque Estadual da Serra do Mar, Núcleo Cunha, 19 Mar. 1996, A. Rapini, et al. 73 (SP); Estação Biológica, Alto da Serra, 800-900 m, 6 Mar. 1929, A. Smith 2076 (BA, NY); Estação Experimental, área nativa, Pariquera-Açu, 2 Apr. 1997, R. B. Torres et al. 182 (IAC); Eldorado, May. 2012, A. Oriani, et al. 450 (ESA); Eldorado Paulista, P.E. Jacupiranga, Núcleo, Caverna do Diabo, Ilha da Caverna, 24³8'91”S, 48²3'31'W, 9 Feb. 1995, Leitão Filho et al. 32980 (UEC); Estação Visconde do Rio Claro, 12 Dec. 1888, A.C.G.G. Loefgren 1220 (SP); Ilha do Cardoso, Jacareu, forest and mangrove swamp, 8 Sep. 1976, P.H. Davis et al. 60747 (UEC); Itapetininga, 9 Feb. 1976, H. F. Leitão Filho et al. 1630 (UEC); Itirapina, Ipiranga, 23 Mar. 1906, A. Usteri s.n. (SP); Juquiá Sitio Areia Dourada, 29 Nov. 1994, K. D. Barreto et al. 3290 (CTES); Paranapiacaba, 16 Jun. 1966, T.M. Pedersen 7795 (CTES, SI); Parque Estadual da Serra do Mar, Núcleo Curucutu, 13 Apr. 2001, L. D. Meireles et al. 151 (UEC); Pinheiros, 8 Jan. 193, A. Gehrt s.n. (IAC); Pindamonhangaba, Fazenda São Sebastião do Ribeirão Grande, noroeste do talhão 10, 22 Feb. 1996, S. A. Nicolau et. al. 1051 (SP); Ponta da Praia, 22 Dec. 1938, E. Guimarães 5 (SP); Rio Claro, 12 Dec 1988, A.C.G.G. Loefgren 11782 (NY); Santo Amaro, Seminário do Espírito Santo, 20 Mar. 1943, L. Roth 10317 (IPA); São Francisco Xavier, Caminho para Cachoeira das Couves, 14 Apr. 1995, J.Y. Tamashiro et al. 902 (UEC); São José dos Campos, Distrito de São Francisco Xavier, 14 Apr. 1995, J.Y. Tamashiro et al. 902 (SP); São Miguel Arcanjo, Parque Estadual Carlos Botelho, 20 Mar. 2002, S. Bortoleto et al. 31 (UEC); São Sebastião, 22 Apr. 2000, J. P. Souza et al. 3398 (UEC); Tapiraí, Reserva Particular da Votorantim, 26 Mar. 2013, C. B. Virillo et al. 8 (UEC).

Taxonomic notes-Until the present, Borreria pterophora has been considered as an imperfectly known but valid name, which is at present day also registered as an endemism of Rio de Janeiro state, Brazil (BFG, 2015), however the examination of the holotype deposited at PR revealed us that is a new synonym of Galianthe palustris.

Galianthe spicata (Miq.) Cabaña Fader \& Dessein, comb. nov. Diodia spicata Miq., Stirp. Surinam. Select. 179-180, t. 52. 1850. Dasycephala spicata (Miq.) Benth. \& Hook. f. ex B.D. 
342 Jacks., Gen. P1. 2: 144. 1873. Borreria spicata (Miq.) Bacigalupo \& E.L. Cabral, Opera Bot. 343 Belg. 7: 307. 1996. Spermacoce spicata (Miq.) Delprete, J. Bot. Res. Inst. Texas 1(2): 1028. 344 2007. TYPE: SURINAM: Sylvarum prope Bergendaal, H.C. Focke s.n. (holotype U!, isotypes 345 HAL0113849!, K000265575!).

346 Diodia denudata Standl., J. Wash. Acad. Sci. 15(5): 105. 1925. Type: PANAMÁ, on wet stream 347 bank along the Río Tapia, near sea level, 24 Dec 1923, P. C. Standley 28123 (holotype: 348 US01154022!).

\section{Description}

350 Herb or subshrub $80-140 \mathrm{~cm}$ alt., erect, stems simple to much branched. Stems quadrangular, 351 fistulose, glabrous, angle weakly alate or without wings, glabrous. Leaves pseudopetiolate, pseudopetiole $0.5-2 \mathrm{~mm}$ long, blades elliptic or narrowly elliptic, papery or subcoriaceous when dry, adaxially glabrescent or scabridous, abaxially glabrous, only scabridous on nerves, base acute or cuneate, apex acute or acuminate, $30-110 \times 10-33 \mathrm{~mm}$; 5-7 secondary nerves, visible on both faces; stipular sheath 1.5-3 mm long, margin truncate or scarcely triangular, pilose, with 5-7 fimbriae, fimbriae 2-7 mm long, with some antrorse hairs. Inflorescences spiciform, partial inflorescences glomeriform, axillary, (5)10-25 per flowering branch, with 3-20 flowers, bracts foliaceous, decreasing in size towards the apex, sometimes up to the same size than the partial inflorescence. Flowers homostylous, calyx 4-lobed, hypanthium obconic, 0.55-6 mm long, puberulous, lobes 0.2-0.3 mm long, unequal, subtriangular, apex acute, margin ciliate; corolla subtubular, slightly expanded to the apex, 1-1.2 mm long, white or greenish white, sometimes with apex of lobes lilac, glabrescent outside, with a ring of moniliform hairs near insertion of the filaments, tube 0.5-0.7 mm long, lobes ovate, apex acute, internally with some scattered hairs, externally pilose and papillose, especially at the apex, 0.2-0.5 mm long, anthers $0.2-0.25 \mathrm{~mm}$ long, oblong, sometimes with a theca visibly smaller than the other, filament $0.15 \mathrm{~mm}$ long; pollen grains 7-zonocolpate, prolato-spheroidal, small, $\mathrm{P}=30.3 \mu \mathrm{m}, \mathrm{E}=28 \mu \mathrm{m}$, colpi long, endoaperture an endocingulum, tectum bireticulate, microreticulate, suprareticulum psilate, infrareticulum with muri nanospinose or psilate; stigma bifid, divided up to the half of its length, with papillae only in the internal face of the stigmatic branches, scarcely exerted. Fruit a capsule, 1.2-1.4 $\times 1-1.2 \mathrm{~mm}$, longitudinally separated from the pedicel upwards up to median portion of the fruit, both mericarps remain attached to each other at the upper part, mericarps indehiscent,

372 subglobose, ventral face flat, slightly laterally compressed, dorsal face pubescent, hispidulous or 
373 glabrescent; seeds $0.8-1 \times 0.35-0.45 \mathrm{~mm}$, oblong or ovate in outline, plane-convex, ventral face 374 with a ample groove, dark brown or nigrescent; exotesta reticulate-foveate, cells polygonal, 375 almost isodiametric. Fig. 4, 5

376 Distribution-Brazil (Amazonas, Roraima, Rondônia, Para, Mato Grosso), Colombia (Chocó), 377 French Guaina, Panamá, Suriname, and Venezuela (Amazonas y Anzoátegui).

378 Ecology_Galianthe spicata grows inside or edges of humid forests.

379 Additional Specimens Examined-BRAZIL: Amazonas, vicinity of Camp Tucano, Rio Tucano, 3803 Dec. 1965, B. Maguire et al. 60319 (IAN, MO); Rondonia, Porto Velho, along hwy 36492 km, 381 by road NE of junction with, 09²2'S 0644 $40^{\prime} \mathrm{W}, 20$ Apr. 1987, H. M. Nee 34960 (MO); Roraima, 382 Dormida, Serra do Lua, foothills of Serra da Lua, 13 Jan. 1969, G. T. Prance 9271 (MO); Pará, 383 Conceição do Araguaia, near Corrego São João and Troncamento Santa Teresa, 8 Feb. 1980, T. C. 384 Plowman 8524 (MO, NY); Altamira Gleba Curuaé, Jul. 2005. M. Sobral et al. 10020 (BHCB). 385 COLOMBIA: Chocó, near Madurex Logging Campn above Teresita and below the rapids on Rio 386 Truando, Feb. 1967, J. A. Duke 9977 (MO); ídem, logging road ca. 2-4km NW of Teresita, 100 387 m, 18 May. 1967, J. A. Duke 11055 (MO). FRENCH GUIANA: Kamakusa, upper Mazaruni 388 River, 23-29 Nov. 1922, J. S. de la Cruz 2808 (MO); Route de l'Est (N2), Montagne Maripa, c. $38931 \mathrm{~km} \mathrm{~S}$ of the Comte bridge, c. selectively logged forest, $04^{\circ} 26^{\prime} \mathrm{N}, 52^{\circ} 20^{\prime} \mathrm{W}, 3$ Dec. $1994, L$. 390 Andersson 1961 (MO).

391 GUYANA: Rupununi, Kanuku Mts., Crabwood Cr. Camp 2 forest, on brown loamy sand, $3^{\circ} 07^{\prime} \mathrm{N}$, $39259^{\circ} 06^{\prime} \mathrm{W}, 260$ m, 2 Apr. 1994, M. J. Jansen-Jacobs et al. 3564 (MO); idem, E Kanuku Mts, NE of 393 Warimure, in forest, $03^{\circ} 05^{\prime} \mathrm{N} 059^{\circ} 20^{\prime} \mathrm{W}, 200-500 \mathrm{~m}, 23$ Jan. 1991, M. J. Jansen-Jacobs et al. 3942189 (MO). PANAMA: Canal Area, Barro Colorado Island, 10-100 m, 909'17"N, 7950'53"W, 39516 Dec. 1967, T. B. Croat 4373 (MO); idem, 99'17"N, 7950'53"W, 18 Mar. 1969, T. B. Croat 3968738 (MO); idem, 99'0"N, 7951'0"W, 1931, S. Aviles s.n. (MO); Cerro Azul, 700 m, 9¹0'2"N, 397 79²4'59"W, 29 Jul. 1972, W.G. D'Arcy 6199 (MO). idem, 700 m, 9¹0'13"N, 79²5'13"W, 7 Jun. 398 1970, A. Kant 46 (MO); Cerro Jefe, 1000 m, 9¹4'0"N, 79²2'0"W, 12 Sep. 1994, C. Galdames et 399 al. 1604 (MO); Colón: Santa Rita, 9²0'0"N, 7947'0"W, 6 Apr. 1969, W. H. Lewis et al. 5238 400 (MO); idem, 9²0'0"N, 7947'0"W, 6 Apr. 1969, W. H. Lewis et al. 5238 (MO); idem, 9²0'0"N, 401 7947'0"W, 6 Apr. 1969, W. H. Lewis et al. 5238 (MO); idem, 9²0'13"N 07946'04"W, 31 Jan. 402 1971, T. B. Croat 13191 (MO); idem, 9¹9'42"N, 7947'27"W, 9 Jul. 1971, T. B. Croat \& M. P. 403 Duncan 15339 (MO); Gamboa Pipeline Road, 90 m, 99'36"N, 7944'44"W, 9 Feb. 1974, M. H. 
404 Nee 9577 (MO); Pipeline Road, 50-100 m, 9¹0'0"N, 7946'0"W, 23 May. 1969, R. L. Lazor 4053464 (MO). SURINAME: 1850, F.A.W. Miquel s.n. (K000265073). Brokopondo: Brownsberg 406 Nature Park, Trail to Mazaroni Val. Primary forest, 0456'N, 55¹1'W, 400-450 m, 24 Jan. 1999, 407 P.G. Delprete 7083 (MO); Marowijne: Nassau Mts, Plataeu C, lateritic rocky soil, 449'N, 408 54³6'W, 500-550 m, 26 Jan. 2003, M.J. Jansen-Jacobs et al. 6254 (MO). VENEZUELA: 409 Amazonas, Atabapo, 5km al Norte de la desembocadura del Rio Orinoco, 2²4'N, 64²4'W, 400 410 m, Oct. 1991, E. Marín 1678 (MO); Anzoátegui, Cabeceras del Morichas Largo, entre Santa 411 Elena y San Pedro a unos 30 km Sur de la Viuda, 9 Nov. 1984, R. A. Montes 2524 (MO).

\section{First record from Peru}

413 Galianthe boliviana E.L. Cabral, Brittonia 57(2): 142, f. 1. 2005. TYPE. BOLIVIA: La Paz: 414 Inqusivi, Cerro Aguada, 2500-2800 m, 22 Nov 1991, M. Lewis 40645 (holotype: LPB0000936!; 415 isotype MO!).

416 Distribution-Sub-Andean foothills of Bolivia and Peru. Galianthe boliviana grows mainly on 417 eroded slopes between 1800 and 3150 m of altitude, especially in open forest of Alnus acuminata 418 Kunth. of Yungas at 2800-3000 m of altitude.

419 Taxonomic notes-Galianthe boliviana is similar to Galianthe dichasia and G. cymosa in having 420 cymoidal inflorescences with subglomeriform partial inflorescence, but differs from these in 421 possessing stems $20-30 \mathrm{~cm}$ tall, with smooth, glabrous, and narrowly winged angles.

Additional Specimens Examined-PERU: Cusco, Quispicanchis, Marcapata, $176 \mathrm{~km}$ from 423 Cusco on road to Maldonado, Marcapata to Cocha, 8 Mar. 1991, 13²5'S 07054'W, 3150 m, 424 Percy Núñez V. \& C. Paycarmayta 13140 (MO).

425 Key to Galianthe species with indehiscent mericarps (modified from Cabral \& Bacigalupo, 1997)

426 1. Stipular sheath tubular, prolonged above the insertion of the corresponding pairs of leaves. .2

428 1'. Stipular sheath truncate, never surpassing the insertion of the corresponding pairs of 429 leaves. 3

2. Stipular sheath pilose; stems with strongly alate angles; Brazil. 
433 2'. Stipular sheath glabrous; stems without wings. $434 \quad$ Brazil ..G. polygonoides E.L. Cabral \& Bacigalupo

4353 . Leaves only with one nerve visible on abaxial face .4

436 3'. Leaves plicate nervose.. .7

4. Inflorescences pauciflorous, in lax cymoid, partial inflorescences 1-florous. Ecuador, Perù G. dichotoma

4'. Inflorescences multiflorous, partial inflorescences multiflorous, in fascicles or glomeruli.... .5

5. Inflorescences cymoid, partial inflorescences glomeriform, calyx 4-lobed, pollen with reticulate exine. Colombia. bogotensis (Kunth) E. L. Cabral \& Bacigalupo

5'. Inflorescences thyrsoid, spiciform, or cymoid, partial inflorescences fasciculate, calyx 2-4lobado, pollen grains with bireticulate exine. 6

6. Inflorescences thyrsoid-spiciform or cymoid, primary axis shorter than the laterals, calyx 2 (4) lobed, corolla of long-styled flowers with a fringe of hairs from apex of anthers to base of tube; pollen grains 6-7 zonocolpate, both reticula complete, fruit 2-3 times wider than long, . brasiliensis (Spreng.) E.L. Cabral \& Bacigalupo

6'. Inflorescences thyrsoid-spiciform, primary axis longer than the laterals, calyx always 4lobed, corolla of the long-styled flowers with ring of hairs, pollen grains 8-10 zonocolpate, suprareticulum incomplete, fruit as long as wide, , Mesoamerica...

456 7'. Partial inflorescences pauciflorous, fasciculate .8

8. Inflorescences spiciform, con 5-20 partial inflorescences per flowering branch. Brazil, Colombia, French Guiana, Panama, Surinam, and Venezuela. G. spicata

8'. Inflorescences thyrsoid or cymoid, with 3-5 partial inflorescences per flowering. 9

$4619 . \quad$ Stems notoriously alate. 10

462 9'. Stems obscurely alate...

463 10. Inflorescences thyrsoid or with a simple axis, partial inflorescences glomeriform, flowers 464 homostylous, calyx 2-3 lobed, corolla 2-3 lobed... 
$46510^{\prime}$. Inflorescences cymoid, partial inflorescences subglomeriform, flowers distylous, calyx 4466 lobed, corolla 4-lobed, Argentina, Brazil, Paraguay, and Uruguay.................. G. dichasia (Sucre \& C.G. Costa)

Galianthe palustris and G. spicata share the same taxonomic and nomenclatural history. First, they were described under Diodia, later they were added to genus Borreria (Bacigalupo \& Cabral, 1996, 1998) due to the presence of homostylous flowers and type of fruit. Later they were, transferred to the genus Spermacoce (Delprete et al., 2005; Delprete, 2007). In 1998, Bacigalupo \& Cabral (1998) observed that G. palustris (then still Borreria palustris) is characterized by a thyrsoid inflorescence that is similar to that of Galianthe. Despite this remarkable observation, the authors decided to transfer the species to genus Borreria. Nearly a decade later, Delprete et al. (2005) and Delprete (2007) transferred both species to Spermacoce in an attempt to create a broad genus concept for Spermacoce. 
496 Despite overall molecular evidence, Galianthe spicata and G. palustris also share similar

497 morphological characteristics with the other Galianthe species (e.g. spiciform and thyrsoid 498 inflorescences, a bifid stigma and pollen grains with a double reticulum). This last character 499 appears in most species of Galianthe, except for G. bogotensis (Kunth) E.L. Cabral \& 500 Bacigalupo, G. dichotoma (Willd. ex Roem. \& Schult.) E. L. Cabral \& Bacigalupo, and the new 501 species G. vasquezii, which have simple reticulum. Pire (1997) hypothesized that in a genus 502 mainly represented by species with double reticulum pollen grains, the simple reticulum is the 503 result of the absence of an infrareticulum persisting only a suprareticulum.

504 Current molecular data indicates that the phylogenetic position of Diodia palustris (Galianthe 505 palustris) and D. spicata (G. spicata) make Galianthe paraphyletic. The Galianthe clade, 506 including both former Diodia species, is strongly supported and has two molecularly well-defined 507 clades. The [Diodia palustris + D. spicata $]+$ G. brasiliensis clade is composed only by species 508 with capsules separating into two indehiscent mericarps and which is a diagnostic character of 509 Galianthe subgen. Ebelia. The sister clade, [G. eupatorioides + G. grandifolia $]+$ G. peruviana, 510 includes species of Galianthe subgen. Galianthe, and is characterized by fruits with dehiscent 511 valves. Both morphological and molecular data support the transfer of two former Diodia species 512 to Galianthe, and more specifically in subgen. Ebelia. Additionally, and according to present 513 sampling, the two subgenera described by Cabral \& Bacigalupo (1997) seem to be monophyletic.

514 The transfer of Diodia spicata to Galianthe was originally proposed by Dessein (2003), based on 515 fruit, polynological and molecular features.

516 Even though morphological and molecular data show that three species share several 517 characteristics with Galianthe subgen. Ebelia, there is a significant difference with the other 518 species of the subgenus. The three species, unlike the remainder, have homostylous flowers. As a 519 result, these results demonstrate the presence of a new floral trait in Galianthe and therefore 520 strongly modify the generic concept of the genus.

521 According to Groeninckx et al. (2009), distyly is often related with double reticulum pollen 522 grains in the tribe Spermacoce. Nevertheless, in the genus Galianthe there are some exceptions to 523 this generalization (e.g. G. bogotensis (distyly and simple reticulum), G. spicata and G. vasquezii 524 (homostyly and double reticulum), and G. palustris (homostyly and simple reticulum). Cabral \& 525 Bacigalupo (1997) mentioned that G. dichotoma presents an intermediate state between 526 distyly/homostyly and pollen with simple reticulum. The authors defined this phenomenon as an 527 "unclear dimorphism" (in Spanish "dimorfismo poco manifiesto"). Future studies are necessary 528 in order to clearly define the floral morphs that are present in these species. 


\section{ACKNOWLEDGMENTS}

We thank the herbarium curators for providing material, especially James Solomon from MO and Rocio Rojas from HOXA. We also thank HOXA's staff; Rodolfo Vasquez and Thania Carhuaricra for sending us images of Galianthe vasquezii's. We also thank Pedro Cuaranta for his help in the illustration of the new species. The second author thanks Beatriz Galati for the selfless assistance in the observation in MEB (UBA) of the pollen grains, and reproductive structures of Galianthe palustris and G. spicata. The third author thanks Charlotte Taylor for the invaluable collaboration.

\section{REFERENCES}

Bacigalupo NM, Cabral EL. 1996. Infrageneric classification of Borreria (RubiaceaeSpermacoceae) on the basis of American species. Opera Botanica Belgica. 7:297-308.

Bacigalupo NM, Cabral EL. 1998. Nota sobre dos especies de Borreria (RubiaceaeSpermacoceae). Hickenia 2:261-266.

Bacigalupo NM, Cabral EL. 1999. Revisión de las especies americanas del género Diodia (Rubiaceae-Spermacoceae). Darwiniana 37:153-155.

Baldwin BG, Markos S. 1998. Phylogenetic utility of the external transcribed spacer (ETS) of 18S-26S rDNA: congruence of ETS and ITS trees of Calycadenia (Compositae). Molecular Phylogenetics and Evolution 10:449-463.

Barker FK, Lutzoni F. 2002. The utility of the incongruence length difference test. Systematic botany 51:625-637.

Cabaña Fader AA, Salas RM, Dessein S, Cabral EL. 2016. Synopsis of Hexasepalum (Rubiaceae), the priority name for Diodella and a new species from Brazil. Systematic botany 41(2):408-422. DOI 10.1600/036364416X691803

Candolle AP. 1830. Diodia. Prodr. 4:563-565. Paris: Treuttel \& Würts.

Cabral EL. 2002. Revisión del género Galianthe Griseb. (Rubiaceae). Doctoral Thesis. Universidad Nacional del Nordeste, Corrientes, Argentina.

Cabral EL, Bacigalupo NM. 1997. Revisión del género Galianthe subgen. Ebelia stat. nov. (Rubiaceae: Spermacoceae). Annals of the Missouri Botanical Garden 84(4): 857-877. 
557

558

559

560

561

562

563

564

565

566

567

Delprete PG, Smith LB, Klein RM. 2005. Rubiáceas, Volume 2 - Gêneros de H-T: 20. Gardênia até 46. Tocoyena. Pp. 349-842 in Flora Ilustrada Catarinense, ed. Reis, A. Itajaí: Herbário Barbosa Rodrigues.

Delprete PG. 2007. New combination and new synonymies in the genus Spermacoce (Rubiaceae) for the Flora of Goiás and Tocantins (Brazil) and the Flora of The Guianas. Journal of the Botanical Research Institute of Texas 1: 1023-1030.

Dessein S. 2003. Systematic Studies in the Spermacoceae (Rubiaceae). Doctoral Thesis. Katholieke Universiteit Leuven, Leuven, Belgium.

Doyle JJ, Doyle JL. 1987. A rapid DNA isolation procedure for small quantities of fresh leaf tissue. Phytochemical Bulletin, Botanical Society of America 19: 11-15.

Erdtman G. 1966. Pollen morphology and Plant Taxonomy Angiosperm. An introduction to Palynology I. Hatner Publishing Company, New York and London.

Farris JS, Kallersjo M, Kluge AG, Bult C. 1995. Testing significance of incongruence. Cladistics 10:315-319.

Flora do Brasil 2020 em construção. Jardim Botânico do Rio de Janeiro. Disponível em: < http://floradobrasil.jbrj.gov.br/ >. Accessed: 05 Dec. 2016.

Google Earth. 2015. Available in <http://www.google earth.com.ar/>. Access in May 2016.

Groeninckx IS, Dessein H, Ochoterena C, Persson TJ, Motley J, Kårehed B, Bremer S, Huysmans, Smets E. 2009a. Phylogeny of the herbaceous tribe Spermacoceae (Rubiaceae) based on plastid DNA data. Annals of the Missouri Botanical Garden. 96: 109-132.

Hijmans RJ. 2013. DIVA-GIS, a geographic information system for the analysis of biodiversity data. Version 7.5. Available in <http://www.diva.gis.org>. Accessed in Feb 2017.

Huelsenbeck JP, Ronquist F. 2001. MRBAYES: Bayesian inference of phylogeny. Bioinformatics 17: 754-755.

IUCN. 2001. IUCN Red List Categories. Gland, Switzerland and Cambridge, Prepared by the IUCN Species Survival Comission. IUCN.

Johnson LA, Soltis DE. 1998. Assessing congruence: empirical examples from molecular data. In: Soltis DE, Soltis PS, Doyle JJ. (Eds.), Molecular Systematics of Plants II DNA Sequencing. Kluwer Academic Publishers, Boston, pp. 297-343.

Katoh K, Misawa K, Kuma K, Miyata T. 2002. MAFFT: a novel method for rapid multiple sequence alignment based on fast Fourier transform. Nucleic Acids Res. 2002;30:3059-3066. 
588

589

590

591

592

593

594

595

596

597

Kirkbride JH. 2014. Hexasepalum teres (Rubiaceae), a new combination. Journal of the Botanical Research Institute of Texas 8(1): 17.

Kirkbride JH, Delprete PG. 2015. New combinations in Hexasepalum (Rubiaceae: Spermacoceae). Journal of the Botanical Research Institute of Texas 9(1): 103-106.

Negrón-Ortiz V, Watson LE. 2002. Molecular phylogeny and biogeography of Erithalis (Rubiaceae), an endemic of the Caribbean Basin. Plant Systematics and Evolution. 234: 7183. DOI: $10.1007 / \mathrm{s} 00606-002-0192-2$

Pire SM. 1997a. Género Galianthe subge. Ebelia (Rubiaceae: Spermacoce): estudios palinológico. Annals of the Missouri Botanical Garden. 84:878-887.

Posada D, Crandall KA. 1998. Modeltest: testing the model of DNA substitution. Bioinformatics 1: 817-818. http://dx.doi.org/10.1093/bioinformatics/17.8.754

Punt W, Hoen PP, Blackmore S, Le Thomas A. 2007. Glossary of pollen spore terminology. Review of Paleobotany and Palynology 143: 1-81.

Rambaut A, Drummond AJ. 2007. Tracer v1.4. Available from: http://beast.bio.ed.ac.uk/Tracer.

Ronquist F, Huelsenbeck JP. 2003. MRBAYES 3: Bayesian phylogenetic inference under $\begin{array}{llll}\text { mixed } & \text { models. } & \text { 1572-1574. }\end{array}$ http://dx.doi.org/10.1093/bioinformatics/btg180

Salas RM, Cabral EL, Viana PL, Dessein S, Janssens S. 2015. Carajasia (Rubiaceae), a new and endangered genus from Carajás mountain range, Pará, Brazil. In: Delprete PG. \& Dessein S. (Eds.), Festschrift volume dedicated to Timothy Motley (1966-2013). Phytotaxa 206: 1429. DOI:10.11646/phytotaxa.206.1.4

Small JK. 1913. The seed-plants growing naturally on the everglade keys and in the adjacente everglades southern peninsular Florida: Rubiaceae. Fl. Miami 177: 200.

Swartz O. 1788. Nova Genera et Species planturam seu Prodromus 30.

Swofford DL, PAUP*. 2003. Phylogenetic analysis using parsimony (*and other methods). Version 4.0b10. Sinauer Associates, Sunderland, Massachusetts.

Walter T. 1788. Diodia in Flora Caroliniana. J. Fraser, London. 1: 87.

White TJ, Bruns T, Lee S, Taylor J. 1990. Amplification and direct sequencing of fungal ribosomal RNA genes for phylogenetics.-In: PCR protocols: a guide to methods and applications (ed. M. Innis, D. Gelfand, J. Sninsky \& T. White). P 315-322. Academic Press, San Diego, California. 


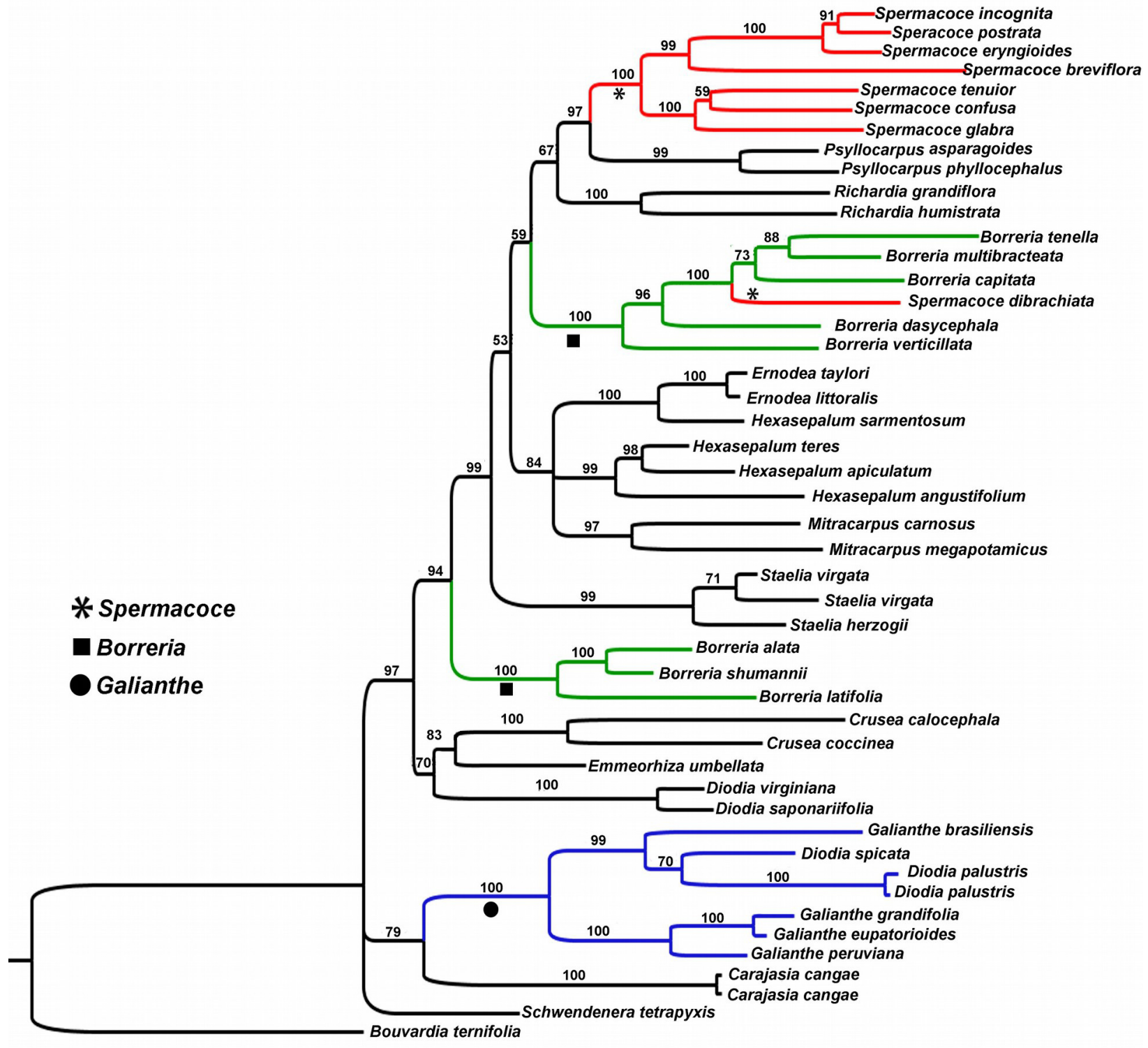


621 Showing the relationship of Galianthe with the remaining genera of Spermacoce clade.

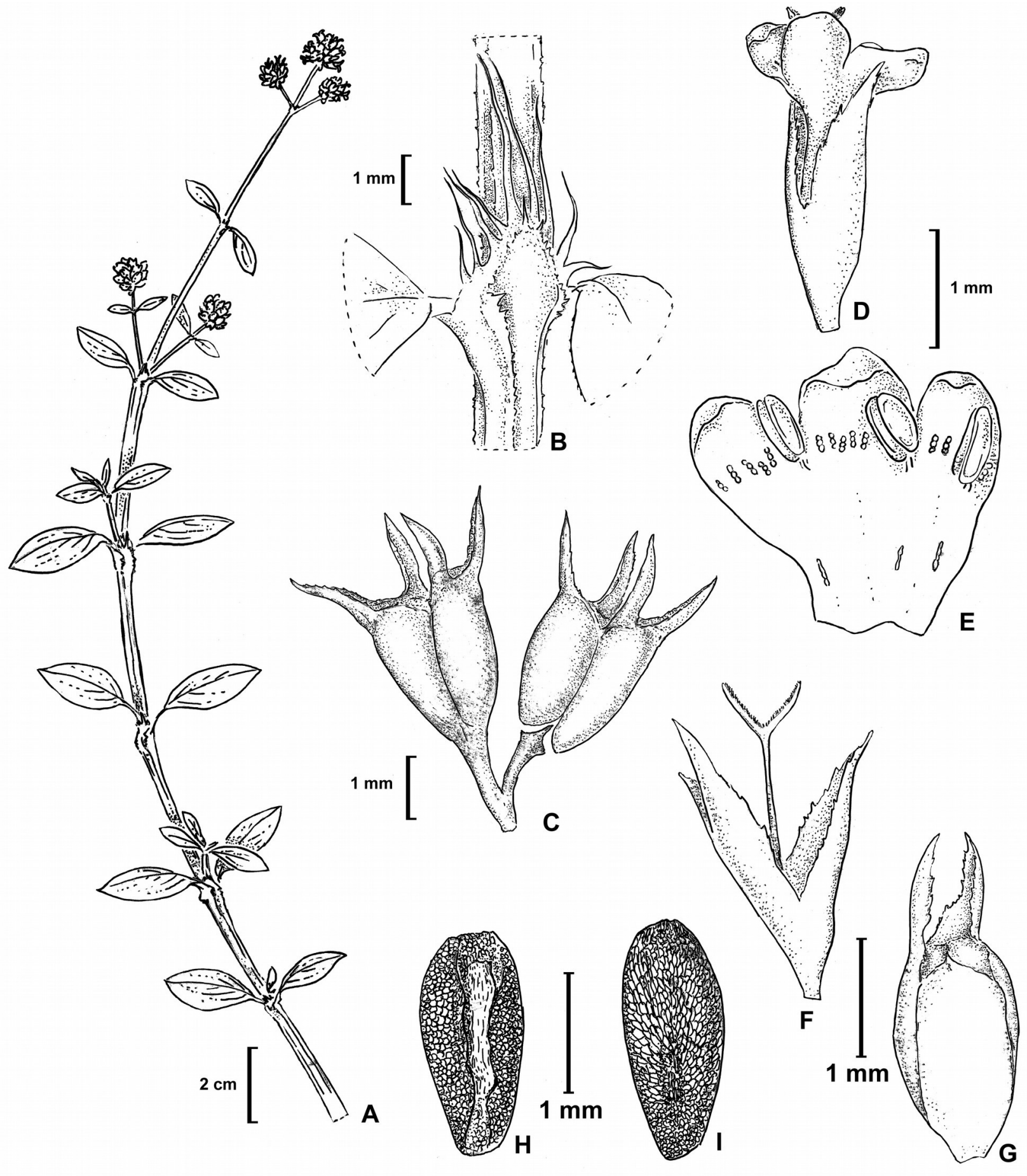


622

623

624

625

Figure 2: Galianthe vasquezii.

23 (A) Apical part of flowering branch. (B) Stipular sheath. (C) Fruit. (D-F) Flower. (E) Inside of corolla (F) Style, stigma and calyx. (G) Ventral view of indehiscent valve, calyx tube and lobes. (H-I) Seeds. (H) Ventral view. (I) Dorsal. All from isotype (MO).
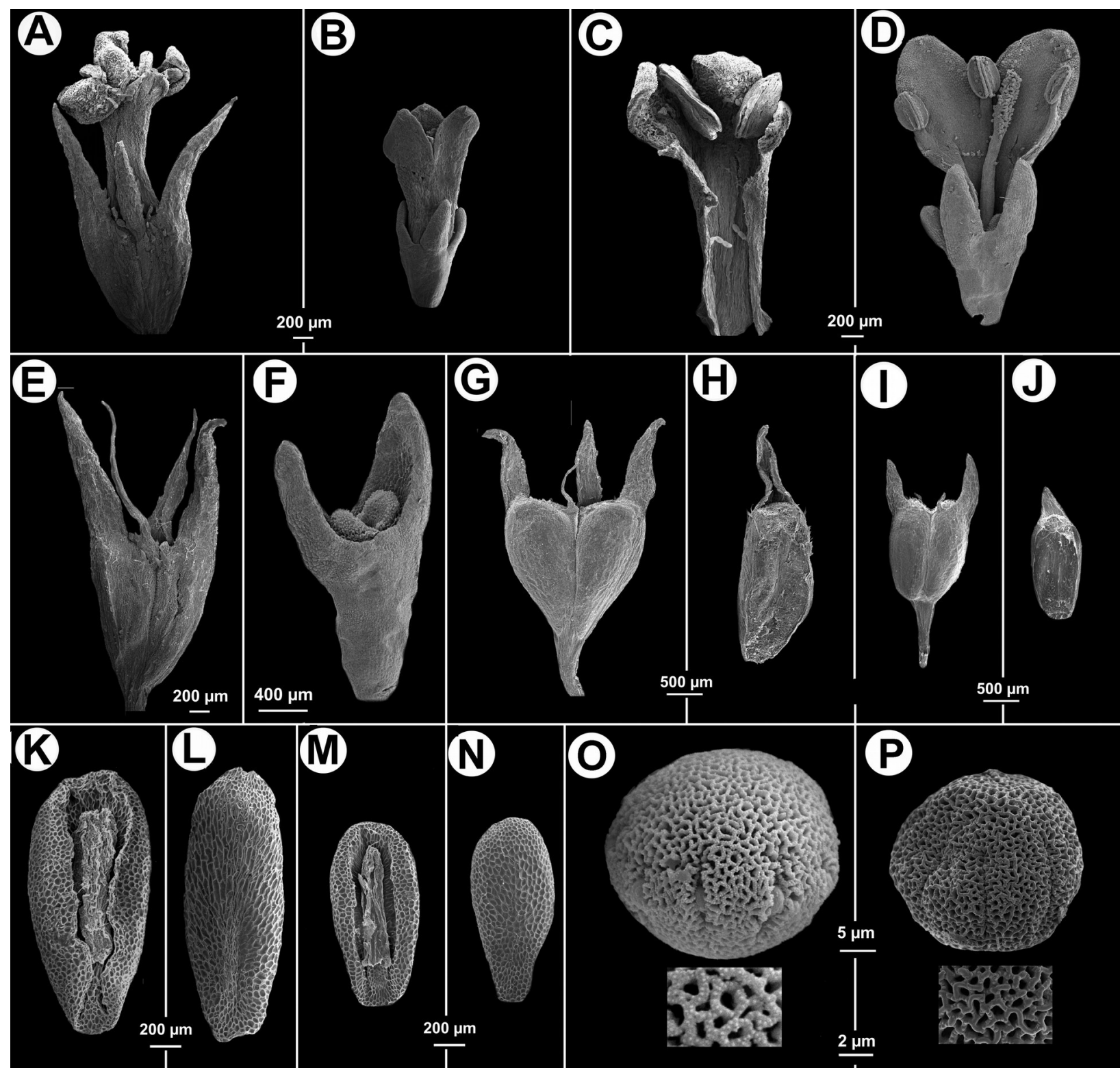

Figure 3: Morphological characters distinguishing Galianthe vasquezii (pictures A, C, E, G, $\mathrm{H}, \mathrm{K}, \mathrm{L}, \mathrm{O}$ from the isotype at MO). and G. palustris (pictures B, D, F, I, J, M, N, P from A. A.

628 Cabaña 19 at CTES). (A) Flower. (C) Inside the corolla with two stamens. (E) Hypanthium and 629 dimorphic calyx lobes. (G) Entire fruit. (H) indehiscent valve. (K) Ventral face of seed. (L) 
630 Dorsal face of seed. (O) Equatorial view of pollen grains, with simple, below a detail of exine.

631 Galianthe palustris. (B) Flower. (D) Opened flower showing inside of corolla and three stamens, 632 style and stigma. (F) Hypanthium, nectariferous disc, and calyx lobes. (I) Entire fruit. (J) 633 indehiscent valve. (M) Ventral face of seed. (N) Dorsal face of seed. (P) Pollen with double 634 reticulum, below a detail of exine showing the suprareticulum incomplete.

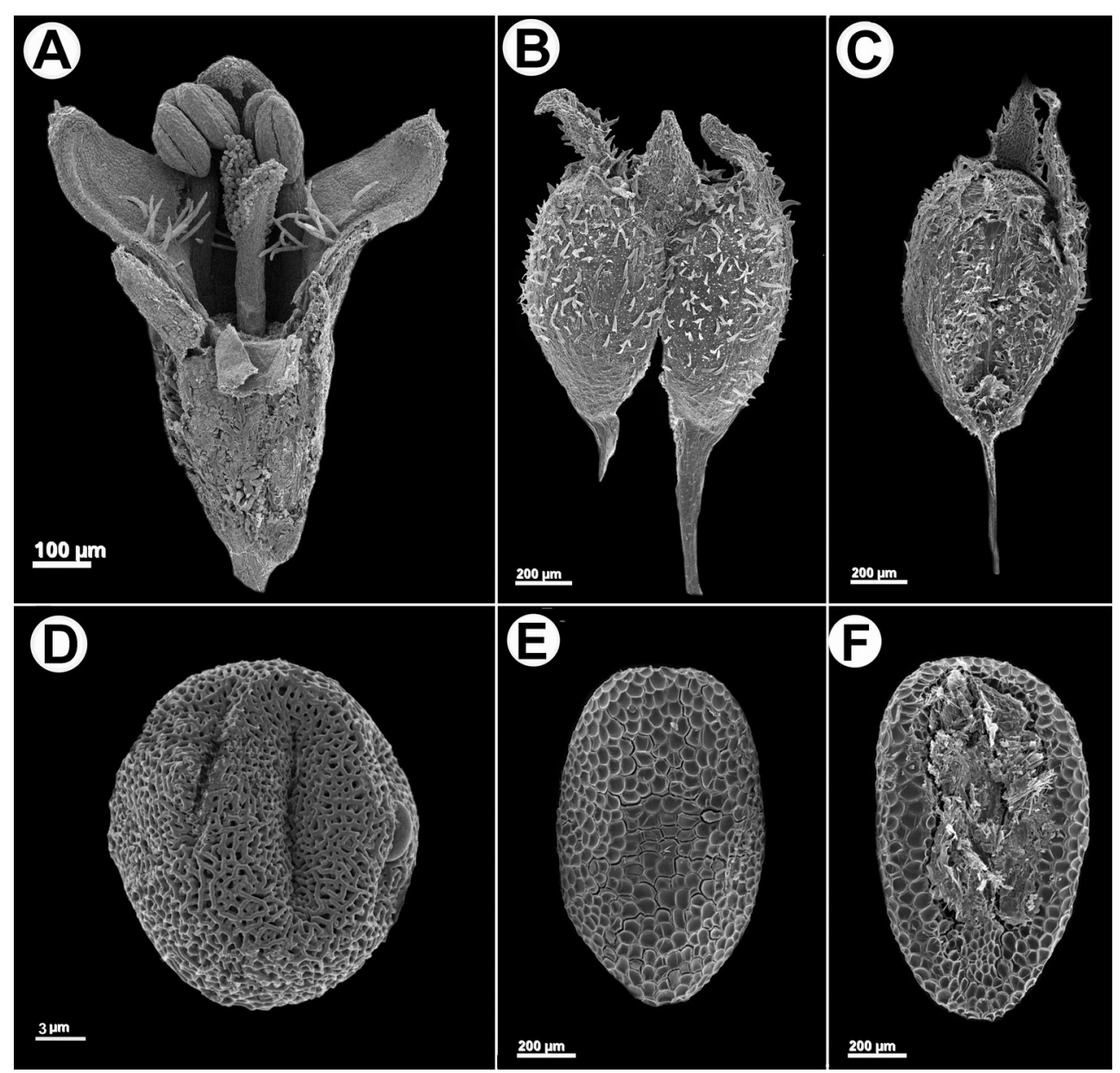

635 Figure 4: Galianthe spicata.

636 (A) Longitudinal section of flower. (B) Fruit with acropetal dehiscence. (C) Indehiscent valve.

637 (D) Equatorial view of pollen grains showing exine with double reticulum. (E) Dorsal face of 638 seed. (F) Ventral face of seed A: From P. G. Delprete 11876 (CAY), B-D: from M. Sobral et al. 63910020 (BHCB). 


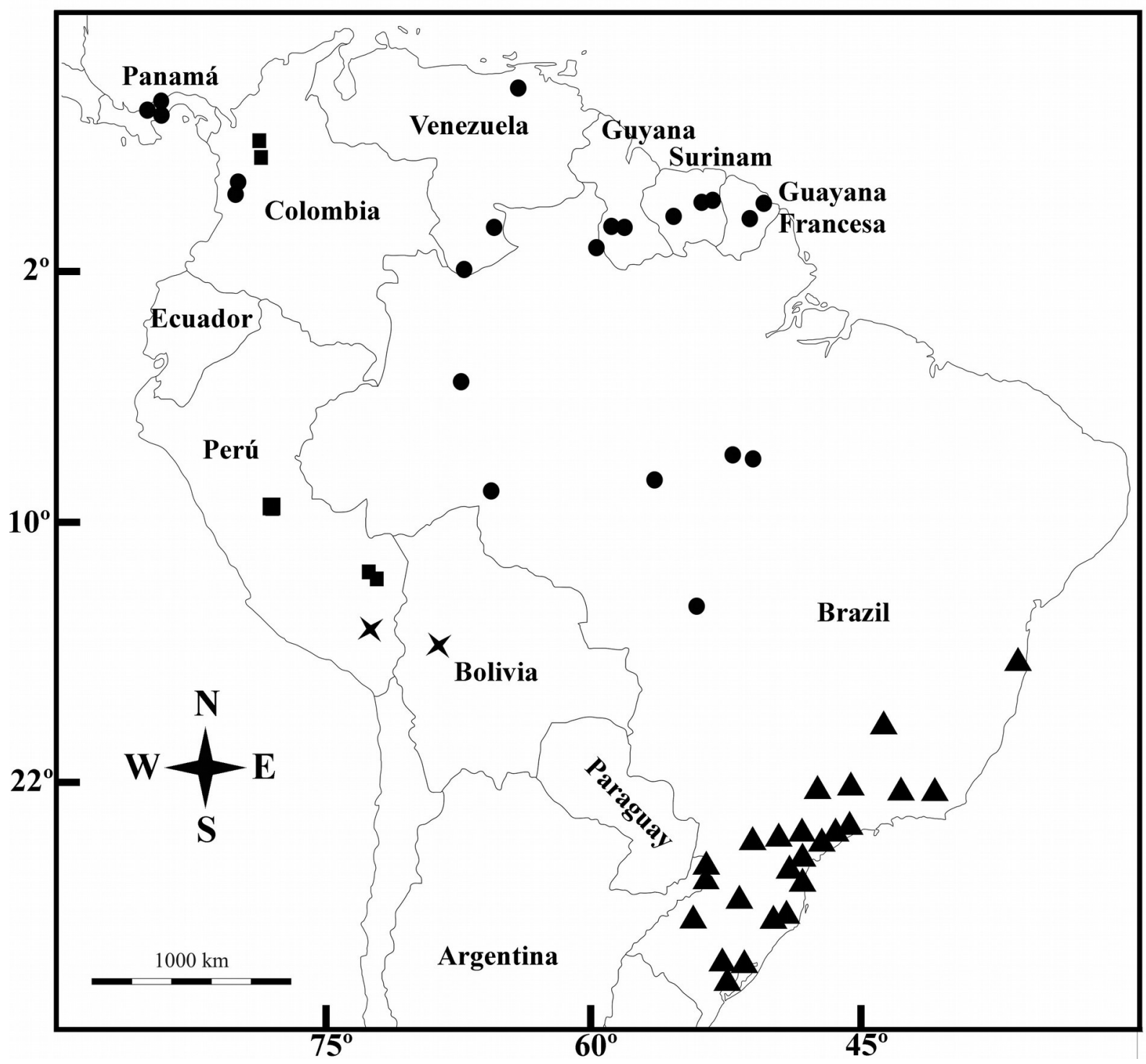

640 Figure 5: Geographic distribution

641 Galianthe boliviana (X), G. palustris (triangle), G. spicata (dots) and G. vasquezii (square). 
643 List of taxa used in the molecular phylogenetic analysis with voucher information (geographical 644 origin, collector, collector number, herbarium, ITS and ETS accession number) and GenBank 645 accession numbers.

646 Ingroup. Borreria G. Mey. B. alata (Aubl.) DC., Brazil, Goiás, Queiroz et al. 14105 (CTES, 647 HUEFS; KF736995, KF737036); B. capitata (Ruiz \& Pav.) DC., Brazil, Bahia, Queiroz et al. 64813688 (CTES, HUEFS; KF736989, KF737031). B. dasycephala (Cham. \& Schltdl.) Bacigalupo $649 \&$ E.L. Cabral, Argentina, Misiones, Salas \& Cabaña 388 (CTES; ITS KF73699); $B$. 650 multibracteata E.L. Cabral \& Bacigalupo. Brazil, Goiás, Queiroz et al. 14261 (CTES, HUEFS; 651 KF736990, KF737032); B. latifolia (Aubl.) K. Schum., Brazil, Goiás, Queiroz et al. 14110 652 (CTES, HUEFS; KF736994, KF737035); B. schumannii (Standl. ex Bacigalupo) E.L. Cabral \& 653 Sobrado, Argentina, Misiones, Cabral et al. 760 (CTES; KF736997, KF737038); B. tenella 654 (Kunth) Cham. \& Schltdl., Brazil, Queiroz et al. 14252 (CTES, HUEFS; KF736988, KF737030); 655 B. verticillata (L.) G. Mey., Argentina, Corrientes, Salas 402 (CTES; KF736998, KF737039); 656 Carajasia, C. cangae, Brazil, Pará, Costa et al 588 (BHCB; KF737015, KF737057); Giorni et al 657179 (BHCB; KF737016, KF737058). Crusea Cham. \& Schltdl., C. calocephala DC., Mexico, 658 Oaxaca, Ochoterena et al. 456 (BR; KF737009, KF737051); C. coccinea DC., Mexico, Oaxaca, 659 Ochoterena et al. 461 (BR; KF737010, KF737052). Diodia L. D. saponariifolia Cham. \& 660 Schltdl., Argentina, Misiones, Cabaña \& Salas 22 (CTES; KF737007, KF737049). D. virginiana 661 L., USA, Missouri, Taylor 12758 (MO; KF737008, KF737050). Emmeorhiza Pohl ex Endl. E. 662 umbellata (Spreng.) K. Schum., Brazil, Bahia, Queiroz et al. 13746 (CTES, HUEFS; KF737000; 663 KF737042). Ernodea Sw. E. littoralis Sw., Cuba, Habana, Rova et al. 2286 (GB; KF737001, 664 KF737043). E. taylori Britton, North Bimini, Correll 44186 (NY; KF737002, KF737044). 665 Galianthe Griseb. G. brasiliensis (Spreng.) E.L. Cabral \& Bacigalupo, Argentina, Misiones, 666 Cabral et al. 758 (CTES; KF737011, KF737053). G. eupatorioides (Cham. \& Schltdl.) E.L. 667 Cabral, Brazil, Goiás, Queiroz et al. 14190 (CTES, HUEFS; KF737012, KF737054). G. 668 grandifolia E.L. Cabral, Brazil, Distrito Federal, Queiroz et al. 14015 (CTES, HUEFS; 669 KF737013, KF737055). G. peruviana (Pers.) E.L. Cabral, Brazil, Minas Gerais, Belo Horizonte, 670 Salas et al. 408 (BHCB, CTES; KF737014, KF737056). G. palustris (Cham. \& Schltd1.) Cabaña 671 Fader \& E. L. Cabral, Verdi et al. 1905 (CTES; MF166824, MF166826); Miguel et al. 19 (CTES; 672 MF166825, MF166827); G. spicata (Miq.) Cabaña Fader \& Dessein, Brazil, French Guiana, 673 Andersson et al. 1961 (GB; AM939535, AM933008); Hexasepalum Bartl. ex DC. H. apiculatum 674 (Willd.) Delprete \& J.H. Kirkbr., Brazil, Bahia. Queiroz et al. 13727 (CTES, HUEFS; KF737003, 675 KF737045). H. angustifolium Bartl. ex DC., Mexico, Rzedowski 17792 (NY; KF737004, 
676 KF737046). H. sarmentosum (Sw.) Delprete \& J.H. Kirkbr., Cameroon, Dessein et al. 1521 (BR; 677 KF737005, KF737047). H. teres (Walter) J.H. Kirkbr., Brazil, Goiás, Queiroz et al. 14089 678 (CTES, HUEFS; KF737048, KF737006). Mitracarpus Zucc. M. carnosus Borhidi \& Lozada679 Pérez, Mexico, Oaxaca, Ochoterena et al. 516 (BR; KF736999, KF737040). M. megapotamicus 680 (Spreng.) Kuntze, Argentina, Corrientes, Salas \& Cabaña 399 (CTES; ETS KF737041). 681 Psyllocarpus Mart. \& Zucc. P. asparagoides Mart. ex Mart. \& Zucc., Brazil, Minas Gerais, 682 Itacambira, Salas et al. 411 (BHCB, CTES; KF737018, KF737060). P. phyllocephallus K. 683 Schum., Brasil, Distrito Federal, Queiroz \& al. 14016 (CTES; ETS KF737061). Richardia L. R. 684 grandiflora (Cham. \& Schltdl.) Steud., Brazil, Bahia, Nova Roma, Queiroz et al. 14055 (CTES, 685 HUEFS; KF737027, KF737066). R. humistrata (Cham. \& Schltdl.) Steud., Argentina, Misiones, 686 Bernardo de Irigoyen, Cabaña \& Salas 17 (CTES; KF737028, KF737067). Schwendenera K. 687 Schum. S. tetrapyxis K. Schum., Brazil, Paraná, Marques et al. 83 (CTES; KF737017, 688 KF737059). Spermacoce L. S. breviflora F. Muell ex Benth., Australia, Harwood 1070 (BR; 689 KF737019, KF737062). S. confuse Rendle, Mexico, Ochoterena et al. 552 (BR; KF737020, 690 KF737063). S. dibrachiata Oliv., Zambia, Dessein et al. 626 (BR; ITS KF737021). S. 691 eryngioides (Cham. \& Schltdl.) Kuntze., Argentina, Salas et al. 378 (CTES; KF736992, 692 KF737033). S. glabra Michx., USA, Missouri, Perry, Taylor 12757 (MO; KF737022, KF73706). 693 S. incognita (E.L. Cabral) Delprete., Brazil, Goiás, Queiroz et al. 14049 (CTES, HUEFS; 694 KF736993, KF737034); S. prostrata Aubl., Brazil, Goiás, Nova Roma, Queiroz et al. 14083 695 (CTES, CTES; KF736996, KF737037); S. tenuior L., México, Novelo et al. s/n (BR; KF737023, 696 KF737065). Staelia Cham. \& Schltdl. S. herzogii (S. Moore) R.M. Salas \& E.L. Cabral, Bolivia, 697 Santa Cruz, Soto et al. 1053 (CTES, USZ; ITS KF737024). S. virgata (Link ex Roem. \& Schult.) 698 K. Schum., Brazil, Bahia, Salas et al. 423 (CTES, HUEFS; ITS KF737025); Brasil, Piauí, Salas 699 et al. 443 (CTES, HUEFS; ITS KF737026). Outgroup. Bouvardia Salisb. B. ternifolia (Cav.) 700 Schltdl., Mexico, Oaxaca, Ochoterena et al. 454 (BR; KF736987, KF737029). 